\title{
Design of the Key Device of Automatic Production Line of Alkaline Noodle
}

\author{
Qiang Yin ${ }^{1, a}$, Feiyu Zhao ${ }^{1, b}$ and Guoquan Zhang ${ }^{1, c}$ \\ ${ }^{1}$ College of Mechanical Engineering, Wuhan Polytechnic University, Wuhan, China \\ ayinqiang@whpu.edu.cn, bydqking1210@sina.com, cgquanzh@sina.com
}

Keywords: Alkaline noodle; Automatic production line; Wrapper cutting and rolling device; Dough wrapper; Roller die

\begin{abstract}
The production technology research of alkaline noodles is of instructive significance to existing noodle industry. As the key device of alkaline noodle automatic production line, the wrapper cutting and rolling device is designed in detail in this paper. On the basis of determining the overall layout of the device and relative position relations between modules, we research the key components, such as dough wrapper conveyer, the fixed-length cutting device, the dough wrapper rolling device, the roller die storage device and the rack, This paper emphasis on the parametric design of wrapper cutting and rolling device, using the MATLAB software to solve the cam profile curve of rising curve of roller die.
\end{abstract}

\section{Introduction}

At present, the noodles on the market can be roughly divided into two kinds: dried noodles and fresh wet noodles. Dried noodle is treated with frying and dehydration process, which makes the taste and nutrition of noodle being heavily discounted. However, fresh wet noodle, without frying, can keep most nutrients. and its taste is relatively good. the characteristics of production technology has significant differences between dried noodle and fresh wet noodle. Especially, the alkaline noodle, as a representative of fresh wet noodle, has a complex production process. which are largely dependent on hand-working. Even there is no specific quantitative standards of technological process of the key working procedure. Thus, according to the specific production process, the suitable automated production line with certain production device should be developed to realize the automatic production of the alkaline noodle.

Japan has been leading the trend in the noodle industry. As the world's largest noodle machinery equipment manufacturers, Japanese TOM LTD. always stands in the forefront of the wet instant noodles equipment, and focus on research and development of the world's top fresh noodle automation devices. Japanese Nissin Food Products Co., Ltd. has developed automatic production lines of the fresh wet noodles, in which the fixed-length cutting and rolling device has played a very important role. There is currently no manufacturer that could develop automatic device of the wrapper fixed-length cutting and rolling for alkaline noodle in the domestic market ${ }^{[1-4]}$.

Common components, such as water purification equipment, mixing equipment, rolling and slitting, packaging equipment, have been relatively mature in production of fresh wet noodle ${ }^{[5,6]}$. But the components like wrapper cutting and rolling device are still relatively simple and backward, mainly by performing simple reforms of the original dry noodle production equipment to produce fresh wet noodle.

Judged from the above facts, we can that China's noodle industry is just getting started and is lacked of the related technology research and development of the equipment, especially for China 's traditional noodles, the study of the industrialized production, is still deficient. components, The production technology research of alkaline noodles is of instructive significance to existing noodle industry .It not only enriched the market supply, meet the needs of the people, and safeguard the people's physical and mental health, but also has a far-reaching significance on promoting alkaline noodles to all parts of the country, and even the international market ${ }^{[7-10]}$. 


\section{Architectural Design}

The Alkaline dough wrapper cutting and rolling device is divided into five modules: the dough wrapper conveyer, the fixed-length cutting device, the dough wrapper rolling device, the storage device for roller die, and the rack, as shown in Fig. 1.

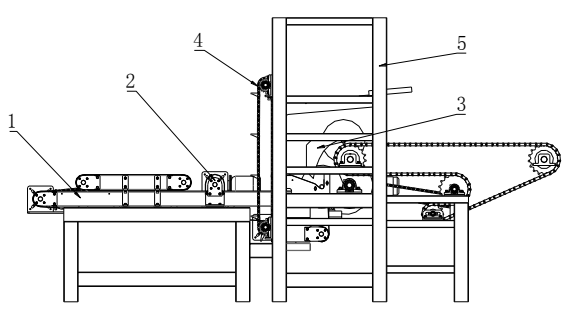

1.the dough wrapper conveyer 2.the fixed-length cutting device 3.the dough wrapper rolling device 4 . the roller die storage device 5 . the rack

Figure 1. Finite Overall layout of the wrapper cutting and rolling device

The general process flow of dough wrapper is introduced at first in this article. The main processes are, (1) press dough wrapper to the thickness of $6 \mathrm{~mm}$ by continuous pressing machine, (2) transfer dough wrapper to the fixed-length cutting device by the dough wrapper conveyer, (3) cut off dough wrapper with a fixed-length under the control of the photoelectric sensor, (4) roll up cut -off dough wrapper to roller die by the dough wrapper rolling device while transferring roller die of the storage device to the dough wrapper rolling device by chain transmission at a fixed frequency, and (5) sent dough wrapper to fermenting box through the chain.

\section{Fixed-length Cutting Device Design}

The function of fixed-length cutting device is to meet the requirement of diameter of individual dough wrapper on the roller die, and cut off dough wrapper continuously transported from the dough wrapper conveyer with a fixed-length. Fixed-length cutting device uses flying shear device as the main actuator of cutting dough wrapper. In order to match well with the roller die storage device, it adopts the photoelectric sensors to detect whether the roller die is in place, and performs the task of cutting off rather than performs cutting mission in fixed time-length.

Fixed-length cutting device consists of motor reducer, cutter shaft, cutter, bearing support of cutter, mounting plate for cutter, and mounting plate for motor reducer panels, as shown in Fig. 2.The cutter is screwed to the cutter shaft with seven screws. In order to compensate for the machining precision and installation accuracy errors, the position of the cutter is adjustable up and down. The specific installation method is shown in Fig. 3.The rotary cutter with single blade shape helps the blade make direct contact with the dough wrapper, so that the cutting can be more effective. In addition, the photoelectric sensors are installed in the roller die storage device at a particular location.

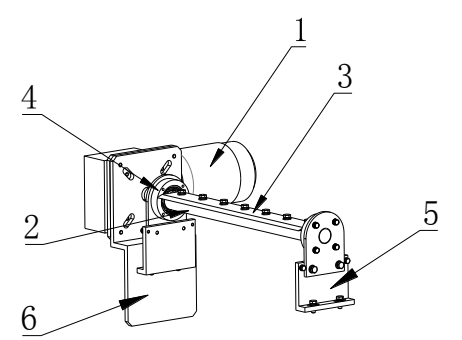

1. motor reducer 2. cutter shaft 3. Cutter 4. bearing support of cutter 5. mounting plate for cutter 6. mounting plate for motor reducer

Figure 2. Finite Fixed-length cutting device 


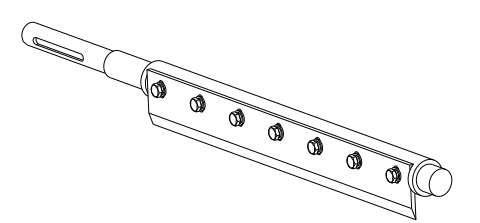

Figure 3. Finite The way of cutter installation

The system is in a state of work, photoelectric sensors installed in the roller die storage device of detect whether the roller die has dropped to a specified height. If so, the feedback signal is provided to the PLC, and then PLC sends execution instructions to the motor driver. The motor drives rotating cutter to cut off the dough wrapper in one cycle of the rotation. The motor will not work if the feedback signal has not been detected.

It should be pointed out that cutting off dough wrapper is most effective when the cutter revolves with the same linear velocity of the belt $v$, namely with $25 \mathrm{~m} / \mathrm{min}$.

\section{Dough wrapper Rolling Device Design}

The dough wrapper rolling device is the most high-tech module among the wrapper cutting and rolling device, and the degree of design difficulty is also very high. Therefore, this section focuses on its design method.

According to the production requirements of equipment, efficiency of rolling wrapper is 100 volumes per hour.

The function of the dough wrapper rolling device is to roll the fixed-length dough wrapper on the roller die. The dough wrapper is sent to the fermentation box for dough recovery after its diameter is up to $300 \mathrm{~mm}$. The device should work smoothly without human intervention from start rolling to finish, with high level automation.

The dough wrapper is rolled on the roller die by successive rotating roller. The roller die climbs along the roller as it is continuously rolled by the dough wrapper. When the dough wrapper is rolled until up to its diameter of $300 \mathrm{~mm}$, at this time, the roller die just climbs to the top of the roller. Then the dough wrapper is taken to the fermentation box by conveyor chain. Climbing trajectory of the roller die is not regular curves. So the irregular curve is is obtained according to actual situation, then limit parts for roller die climbing is designed based on the irregular curve.

Roller Size Determination. The dough wrapper, with a thickness of $6 \mathrm{~mm}$ and a width of $450 \mathrm{~mm}$, can enter the fermentation box only after its wrapped diameter is up to $300 \mathrm{~mm}$. If the roller diameter is less than $300 \mathrm{~m}$, it is possible that the roller die has climbed to the top of the roller, but its wrapped diameter is not yet up to $300 \mathrm{~mm}$. So the roller diameter must be greater than or equal to $300 \mathrm{~mm}$.

Considering flexibility of dough wrapper, there is possible mutual extrusion between the dough wrappers in the process of rolling. Although the length of dough wrapper has reached the theoretical length, the actual rolling diameter has not yet reached $300 \mathrm{~mm}$. Therefore, the roller diameter $d_{r}$ must be appropriately enlarged and initially set to $325 \mathrm{~mm}$, and the roller width $b_{w}$ is initially set to $455 \mathrm{~mm} . d_{r}$ and $b_{w}$ are defined as follows:

\section{$d_{r}=325 \mathrm{~mm}, b_{w}=455 \mathrm{~mm}$}

Theoretical Length of Rolling Wrapper and Rolling Time. The template is used to format your paper and style the text. All margins, column widths, line spaces, and text fonts are prescribed; please do not alter them. You may note peculiarities. For example, the head margin in this template measures proportionately more than is customary. This measurement and others are deliberate, using specifications that anticipate your paper as one part of the entire proceedings, and not as an independent document. Please do not revise any of the current designations.

The width $B$ and thickness $h$ of dough wrapper are $450 \mathrm{~mm}$ and $6 \mathrm{~mm}$.the rolling diameter $D$ of dough wrapper is $300 \mathrm{~mm}$ and the roller die inner shaft diameter $d$ is $50 \mathrm{~mm}$ without consideration of flexibility and mutual extrusion of dough wrapper. the theoretical rolling length of dough wrapper is set as $L$. 
When the dough wrapper is rolled on the roller die, its volume $V$ is defined as follows:

$$
V=\pi\left(\frac{D}{2}\right)^{2} B-\pi\left(\frac{d}{2}\right)^{2} B
$$

When the dough wrapper spread, its volume $v$ is defined as follows:

$$
v=L B h
$$

And combination of two equations is:

$$
\pi\left(\frac{D}{2}\right)^{2} B-\pi\left(\frac{d}{2}\right)^{2} B=\mathrm{LB} h
$$

The theoretical rolling length $L$ of dough wrapper is derived as follows:

$$
L=11454 \mathrm{~mm}=11.454 \mathrm{~m}
$$

The conveyor belt is moving with dough wrapper at a constant speed, and there is no relative movement between them. and thus every time consuming $t$ of rolling dough wrapper can be calculated.

$$
t=\frac{L}{v}=\frac{11.454 m}{25 m / \min }=27.5 \mathrm{~s}
$$

\section{Rising Curve of Roller die}

In analogy with the reversal method of cam design, The protruding part of roller die outer shaft is the equivalent of the roller of disc cam with pushing bar of roller of linear motion along the axis, and its axis trajectory is just the theory profile of disc cam. Therefore, the roller die rising curve can be

\begin{tabular}{|c|c|c|c|c|c|}
\hline Motion Law & Load & Speed & $\begin{array}{|ll|}\underset{(\mathrm{m} / \mathrm{s})}{\text { Max }} & \text { Speed } \\
\end{array}$ & $\begin{array}{l}\text { Max } \\
\text { Acceleration }\left(\mathrm{m} / \mathrm{s}^{2}\right)\end{array}$ & Impact \\
\hline $\begin{array}{l}\text { Isokinetic } \\
\text { motion }\end{array}$ & light & low & 1.00 & $\infty$ & rigidity \\
\hline $\begin{array}{l}\text { Constant- } \\
\text { deceleration motion }\end{array}$ & light & medium & 2.00 & 4.00 & flexibility \\
\hline $\begin{array}{l}\text { Cosine acceleration } \\
\text { motion }\end{array}$ & $\begin{array}{l}\text { mediu } \\
\mathrm{m}\end{array}$ & medium & 1.57 & 4.93 & flexibility \\
\hline $\begin{array}{l}\text { Sine acceleration } \\
\text { motion }\end{array}$ & light & high & 2.00 & 6.28 & non \\
\hline
\end{tabular}
designed according to the related design theory of cam profiles.

First, it is necessary to determine The motion law of pushing bar. The property values and applicable occasions of various kinds of the motion law are shown in Table 1.

Table 1 The property values and applicable occasions of several motion law

Because the beginning and the end of rolling are the key nodes of the movement, the sine acceleration motion law is proposed to ensure no-impact during the movement. The motion equation of Sine acceleration motion law be expressed as:

In the rise travel: 


$$
\left\{\begin{array}{l}
\mathrm{s}=\mathrm{h}\left[\left(\delta / \delta_{0}\right)-\sin \left(2 \pi \delta / \delta_{0}\right) /(2 \pi)\right] \\
\mathrm{v}=\mathrm{h} \omega\left[1-\cos \left(2 \pi \delta / \delta_{0}\right)\right] / \delta_{0} \\
\mathrm{a}=2 \pi \mathrm{h} \omega^{2} \sin \left(2 \pi \delta / \delta_{0}\right) / \delta_{0}{ }^{2}
\end{array}\right.
$$

In the return travel:

$$
\left\{\begin{array}{l}
\mathrm{s}=\mathrm{h}\left[1-\left(\delta / \delta_{0}{ }^{\prime}\right)+\sin \left(2 \pi \delta / \delta_{0}{ }^{\prime}\right) /(2 \pi)\right] \\
\mathrm{v}=\mathrm{h} \omega\left[\cos \left(2 \pi \delta / \delta_{0}{ }^{\prime}\right)-1\right] / \delta_{0}{ }^{\prime} \\
\mathrm{a}=-2 \pi \mathrm{h} \omega^{2} \sin \left(2 \pi \delta / \delta_{0}{ }^{\prime}\right) / \delta_{0}{ }^{\prime 2}
\end{array}\right.
$$

Where, $h$ is the stroke of pushing bar, $\delta$ is the cam angle, $\delta_{0}$ is the motion angle for rise travel, $\delta_{0}{ }^{\prime}$ is the motion angle for return travel, and $\omega$ is the cam angular velocity.

Second, the geometric parameters and the movement parameters of the cam must be determined, and the dough wrapper rolling model is shown in Fig. 4, and the relative position relationship of roller, belt and roller die inner shaft are also shown in Figure 4.During the initial rolling phase, roller die inner shaft scrolls up along rolling plate of roller die from position A to position B.When the roller die moves to position $\mathrm{B}$, its movement enter a the rise travel phase with sine acceleration motion law. The distance between axis line of roller die and roller is $241.5 \mathrm{~mm}$, that is the basic circle radius of cam theoretical contour. When the line between roller die axis and roll axis is perpendicular with the horizontal plane, the angular motion parameters of rise travel is about $81^{\circ}$, the contour displacement of cam is $71 \mathrm{~mm}$.

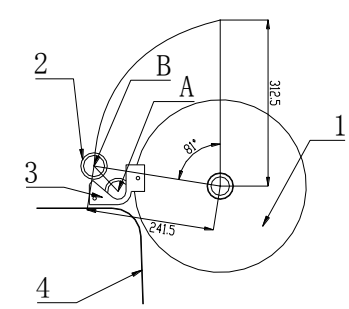

1.roller 2.roller die inner shaft 3. rolling plate of roller die 4.belt

Figure 4. Finite Dough wrapper rolling model

Based on the above analysis, it is concluded that the following geometrical parameters:

(1)cam angle for rise $\delta_{01}=81^{\circ}$;

(2)cam angle for outer dwell $\delta_{02}=99^{\circ}$;

(3)cam angle for return $\delta_{03}=81^{\circ}$;

(4)cam angle for inner dwell $\delta_{04}=99^{\circ}$;

(5)height of lift $h=71 \mathrm{~mm}$;

(6)basic circle radius $r_{0}=241.5 \mathrm{~mm}$;

(7)roller radius $r=15 \mathrm{~mm}$.

Based on every consumption time $t$ of rolling dough wrapper, the CAM rotation angular velocity $\omega$ can be calculated. When rolling dough wrapper, the distance through which roller die rolls up from bottom to the top of roller is the equivalent of cam rise. Therefore, the equations are expressed as:

$$
\frac{t}{360^{\circ}}=\frac{27.5}{81^{\circ}} \Rightarrow t=122.22 \mathrm{~s}
$$

That is,cam takes 122.22 s to rotate every turn, So the speed $n$ of cam is:

$$
\mathrm{n}=1 / \mathrm{t}=8.18 \times 10-3 \mathrm{r} / \mathrm{s} \approx 0.49 \mathrm{rpm}
$$


The angular velocity $\omega$ of cam is:

$$
\omega=\frac{\pi n}{30}=0.0513 \mathrm{rad} / \mathrm{s}
$$

Then, according to the geometric and kinematic parameters, the theoretical contour and motion curve of cam are generated by using MATLAB software programming.

After getting the theoretical contour of cam, the contour curve and displacement motion diagram can be further obtained. Here, the analytical method formula of cam design should be applied. The Cartesian coordinate formulas of contour point are shown as follows:

$$
\left\{\begin{array}{l}
\mathrm{x}=\left(\mathrm{s}_{0}+\mathrm{s}\right) \sin \delta+\mathrm{e} \cos \delta \\
\mathrm{y}=\left(\mathrm{s}_{0}+\mathrm{s}\right) \cos \delta-\mathrm{e} \sin \delta
\end{array}\right.
$$

Among e refers to offset distance, $s_{0}$ refers to basic circle radius of cam, $s$ refers to displacement of push rod, $\delta$ refers to cam angle.

According to this formula, $\mathrm{M}$ files of contour curve are compiled. After running this program.by using MATLAB, cam real profile and displacement motion diagram can be obtained. Cam actual profile of sinusoidal acceleration motion law is shown in Fig. 5, and Cam displacement motion diagram of sinusoidal acceleration motion law is shown in Fig. 6.

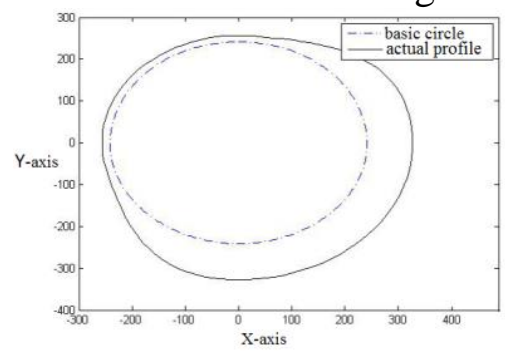

Figure 5. Finite Cam actual profile of sinusoidal acceleration motion law

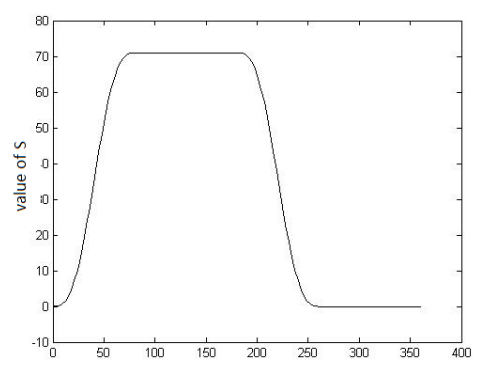

Figure 6. Finite Cam displacement motion diagram of sinusoidal acceleration motion law

It can be seen from Fig . 6, the curve is round and smooth at motion nodes, showing that continuity on the first derivation and the second derivation of displacement function at motion nodes has not sudden change of velocity and acceleration. The motion without rigid impact and flexible impact has reached design requirements.

Calculate the matrix transpose of a given matrix characterizing the actual coordinates of the cam profile. in this $\mathrm{M}$ file, and copy all point coordinates to notepad document to make coordinate file, as shown in Fig. 7. 


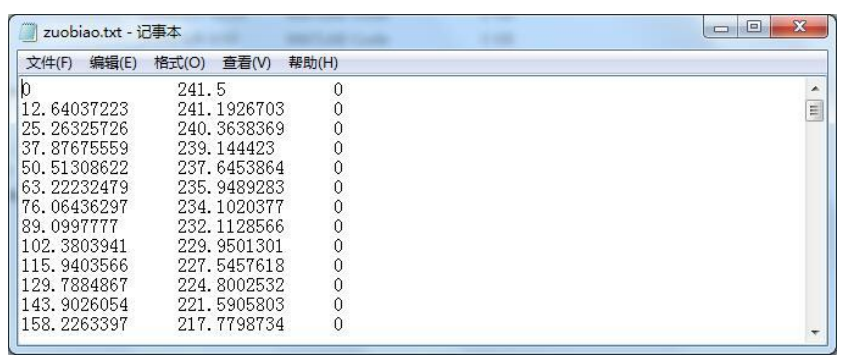

Figure 7. Finite The actual coordinates of the cam profile

Import the coordinate file to SolidWorks software, then generated the cam profile curve. Because just only the rise travel of cam is adopted in this project, the rise travel curve of cam profile curve is intercepted and modified to get the three-dimensional model of the limit climbing part of roller die, as shown in Fig. 8.

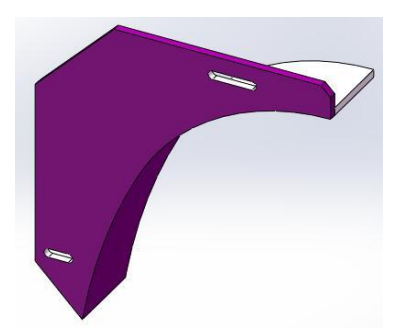

Figure 8. Finite Limit climbing part of roller die

\section{Conclusions}

As the key device of alkaline noodle automatic production line, the wrapper cutting and rolling device is designed in detail in this paper. First, the overall layout of the device and relative position relations between modules is determined. Secondly, the key components, such as dough wrapper conveyer, the fixed-length cutting device, the dough wrapper rolling device, the roller die storage device and the rack, are researched. Finally, This paper emphasis on the parametric design of wrapper cutting and rolling device, using the MATLAB software to solve the cam profile curve of rising curve of roller die.

\section{Acknowledgment}

This research was supported by Educational Commission of Hubei Province of China (D20161706 ). The research reported in the paper was also supported by Foundation of Wuhan Polytechnic University (No.2017y02).

\section{References}

[1] Fu, B X, Assefaw, E G, Sarkar, A K, Carson, G R-Cereal, Evaluation of durum wheat fine flour for alkaline noodle processing, cereal foods world,2006,51(4),178-183.

[2] M Chakraborty, GA Hareland, FA Manthey, LR Berglund, Evaluating quality of yellow alkaline noodles made from mechanically abraded sprouted wheat, Journal of the Science of Food \& Agriculture, 2003 , 83 (5):487-495.

[3] BX Fu,Asian noodles: History, classification, raw materials, and processing, Food Research International,2008,41(9):888-902.

[4] Yu-Chan Huang,His-Mei Lai. Noodle quality affected by different cereal starches[J].Journal of Food Engineering,2010,972:135 143. 
[5] LIU Guo-feng, XU Xue-meng, WANG De-dong, The production and packaging technology of wet raw noodles, packaging engineering,2004,25(4):144-146.

[6] WANG Shan,LIU Xin-ran, LI Qin-yuan, SHEN Qun, CHEN Yan-hui, Study on the production of the wet instant noodle by the technology of high hydrostatic pressure, Science and Technology of Food Industry,2016,37(3): 229-233.

[7] Lin Yantao, The present situation and development trend of instant noodles industry at home and abroad, Grain Science and Technology and Economy,2014,39 (2):66-68.

[8] Sun Dinghong,Chen Jie,Study on the development situation and tendency of international instant noodles industry,Journal of Anhui Agricultural Sciences,2010,38 (36):592-594.

[9] Guo-quan Zhang, Packaging machinery design [M]. Beijing: the printing industry press, 2013.

[10] XIA Yu,Design on production process and key devices of the wet-fresh noodle[D],Central South University of Forestry and Technology,2014.

[11] Dong-ping He, Food factory design [M]. Beijing: China light industry press, 2009.

[12] Liu Rui, Ren Xiaolong, Xing Yanan, et al.Effect of vacuum mixing on the noodles quality properties and optimization of its process parameters, Journal of the Chinese Cereals and Oils Association, 2015,30(9):6-12. 